\title{
Dynamic sedimentation of linear micellar aggregates in a centrifugal field
}

\author{
Alexander Duyndam and Theo Odijk \\ Department of Polymer Technology, Faculty of Chemical Engineering and Materials Science, Delft \\ University of Technology, P.O. Box 5045, 2600 GA Delft, The Netherlands
}

(Received 12 October 1993; accepted 6 November 1993)

\begin{abstract}
We formulate an equation for the dynamic sedimentation of linear micelles (both rodlike and flexible) in the limit of local thermodynamic equilibrium when the recombination and scission of micelles are fast processes. A self-similar solution of the first kind is proposed for rodlike micelles in a free-draining approximation. The analytical structure of the associated nonlinear ordinary differential equation is very different from that pertaining to solutions of nonaggregating molecules. The theory may be of use in an experimental determination of the growth parameters by ultracentrifugation.
\end{abstract}

\section{INTRODUCTION}

There are several reasons for studying the dynamic sedimentation of linear micelles theoretically. Ultracentrifugation may offer a convenient means of characterizing the growth parameters with little ambiguity ${ }^{1}$ (although the use of the ultracentrifuge for characterization purposes is on the decline, worldwide). Light scattering, for instance, has the drawback of our having to know the distribution of the micellar size explicitly. Gravity and centrifugal fields are pure potentials so it is straightforward to develop a transparent theory in terms of a local chemical potential of a surfactant molecule. Finally, because diffusion and sedimentation may occur on well-separated time scales, one expects to be able to simplify the mathematics of the micellar transport to a considerable extent.

The connection between the exchange kinetics of the micelles (i.e., their recombination and breaking apart) and their polymerlike motion was first addressed quantitatively by Cates for entangled solutions. ${ }^{2} \mathrm{~A}$ connection exists even when the solution is dilute: an external field affects the distribution of micellar size which itself has an impact on the transport properties; continuity equations yield a selfconsistent theory for the distribution. Such a program was carried out by several theoretical groups for shear and elongational flow $^{3-7}$ and diffusion. ${ }^{8}$ In an ultracentrifuge experiment, both diffusion and sedimentation will, in the long run, be slow processes compared to the micellar kinetics. It thus make sense to study, in particular, the "fast reaction" regime. Hence, our starting equations are based on arguments similar to those introduced by Cates $e t a l^{3,8}$

We first summarize the usual, linear theory of the sedimentation of nonaggregating molecules, not only for the sake of comparison but also because we present a new method of deriving a similarity solution to the Lamm equation. This is readily extendible to a certain class of nonlinear transport equations. Next, we formulate the continuity equation for the surfactant concentration in the limit of fast exchange or local thermodynamic equilibrium, and evaluate explicit forms for both flexible and rodlike micelles. Here, our interest is especially in the latter which are currently the subject of active investigation. ${ }^{9,10}$ We seek a self-similar solution for the temporal and spatial dependence of the concentration front for rodlike aggregates, albeit in a free-draining approximation. We study the associated ordinary differential equation in some detail.

\section{SEDIMENTATION OF NONAGGREGATING PARTICLES}

We first recall the classical approach to linear sedimentation equations. ${ }^{11,12}$ The Lamm equation describes the time evolution of the concentration profile for a dilute, incompressible solution of noninteracting Brownian particles in a centrifugal field

$$
\frac{\partial X}{\partial t}=\frac{1}{r} \frac{\partial}{\partial r}\left(\frac{k_{B} T}{f} \frac{\partial X}{\partial r}-\frac{m \omega^{2} r^{2}}{f} X\right),
$$

where $X(r, t)$ designates the mole fraction of solute at a distance $r$ from the axis of rotation at time $t, k_{B} T$ the Boltzmann constant times the absolute temperature, $\omega$ the angular velocity, $f$ the translational friction coefficient, and $m$ the mass of a solute particle corrected for buoyancy (see Fig. 1). A common choice for the initial condition is a step function profile at $t=0$

$$
\begin{aligned}
& X(r, t=0)=0 \quad \text { for } r<r_{0}, \\
& X(r, t=0)=X_{0} \quad \text { for } r \geqslant r_{0} .
\end{aligned}
$$

The boundary conditions are

$$
\frac{k_{B} T}{m \omega^{2} r} \frac{\partial X}{\partial r}=X \quad \text { at } r=r_{1} \quad \text { and } r=r_{2} ; \quad t>0,
$$

stating that particles cannot cross the meniscus of the solution or the bottom of the cell, respectively. An exact solution of Eqs. (2.1)-(2.3) has been provided by Archibald in terms of an infinite series of confluent hypergeometric functions. ${ }^{13}$

A convenient, closed approximation can be obtained when we restrict ourselves to the early stages of the sedimentation process. Three regions can then be distinguished: a small one at the bottom of the cell where the solute accumulates, a relatively broad intermediate region virtually unaffected by the two boundaries, and another small region at the top of the cell (see Fig. 2). In the intermediate region, the solute sediments almost as if in an infinite cell. Faxén solved the Lamm equation in this case 


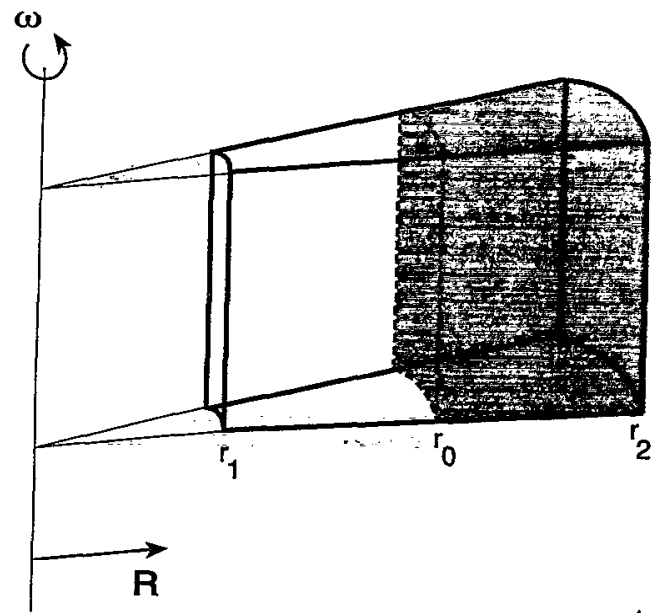

FIG. 1. Cross section of the ultracentrifuge cell, $r_{0}$ denoting the position of the meniscus of the solution.

exactly in 1929. Rewriting Eq. (2.1) in terms of the dimensionless variables: $\Theta(x, t) \equiv X(r, t) / X_{0} ; x \equiv\left(r / r_{0}\right)^{2}$; $\tau \equiv\left(2 m \omega^{2} t\right) / f$ and $\epsilon \equiv\left(2 k_{B} T\right) /\left(m \omega^{2} r_{0}^{2}\right)$

$$
\frac{\partial \Theta}{\partial \tau}=\frac{\partial}{\partial x}\left(\epsilon x \frac{\partial \Theta}{\partial x}-x \Theta\right)
$$

We can express the asymptotic form of his solution as ${ }^{11}$

$$
\Theta=\frac{e^{-\tau}}{2}\left[1-\operatorname{erf}\left(\frac{1-\sqrt{x e^{-\tau}}}{\sqrt{\epsilon\left(1-e^{-\tau}\right)}}\right)+O\left(\epsilon^{1 / 2} \tau^{1 / 2}\right)\right]
$$

Here, $x=O(1), \tau \leqslant O(1)$, and $\epsilon \ll 1$ which define an experimentally realistic limit. The error function is given by

$$
\text { erf } x^{\prime} \equiv \frac{2}{\sqrt{ } \pi} \int_{0}^{x^{\prime}} e^{-y^{2}} d y
$$

The argumentation sketched above depends explicitly on the linearity of Eq. (2.4). We now present a physically motivated derivation of Eq. (2.5) which makes use of the smallness of $\epsilon$ from the beginning. It has the advantage of being applicable to the nonlinear case when the diffusion has a power law dependence on the concentration as in the next section. For small $\epsilon$, we expect the solution to Eq. (2.4) to be a propagating concentration wave whose front is a relatively thin, diffusive boundary layer. This motivates the substitutions $\eta(x, \tau) \equiv x \Theta(x, \tau)$ and $z \equiv \log x$ in Eq. (2.4)

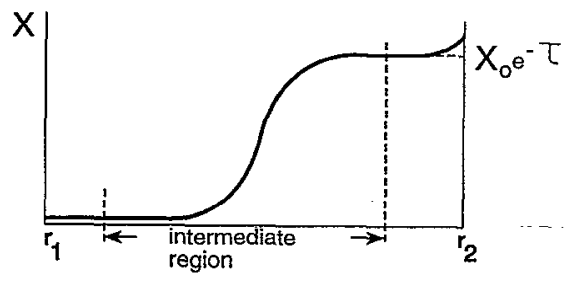

FIG. 2. Schematic plot of the concentration within the cell.

$$
\frac{\partial \eta}{\partial \tau}=\epsilon \frac{\partial^{2}\left(e^{-z} \eta\right)}{\partial z^{2}}-\frac{\partial \eta}{\partial z}
$$

and subsequent elimination of the translating wave by postulating that $\eta(z, \tau)$ has the form $\eta(z, \tau) \equiv \Phi(z$ $-\tau, \tau) \equiv \Phi(y, \tau)$ with $y \equiv z-\tau$

$$
\frac{\partial \Phi}{\partial \tau}=\epsilon \frac{\partial^{2}\left(e^{-y-\tau} \Phi\right)}{\partial y^{2}}
$$

Hence, this equation focuses on the diffusion within the boundary layer. Eliminating the time dependence on the right-hand side by choosing a new time variable $q \equiv 1$ $-e^{-\tau}$, we get

$$
\frac{\partial \dot{\Phi}}{\partial q}=\epsilon \frac{\partial^{2}\left(e^{-y} \Phi\right)}{\partial y^{2}}
$$

Keeping in mind that $\epsilon \ll 1$, we may view the cell as effectively infinite in extent, in the beginning and even in the intermediate stages of the sedimentation. Moreover, well beyond the thin boundary layer, the original variable $\Theta$ decreases as $e^{-\tau}$ in the bottom part of the cell in view of mass conservation. Accordingly, the solution to Eq. (2.8) has to satisfy the effective boundary conditions: $\Phi(y=$ $-\infty, q)=0$ and $\Phi(y=+\infty, q)=1$ besides the initial condition $\Phi(y, q=0)=0$ for $y<0$ and $\Phi(y, q=0)=1$ for $y \geqslant 0$. In fact, to zero order we have $x=e^{\tau+y} \simeq e^{\tau}$ or $\Phi \simeq e^{\tau} \Theta$ for relevant values of $y$ throughout the diffusive layer since Eq. (2.8) shows that $|y|=O\left(\epsilon^{1 / 2} q^{1 / 2}\right) \quad(q \leqslant 1$ so $|y| \ll 1$ at all times if $\epsilon \ll 1$ ). Note that the right-hand side of Eq. (2.8) is greatly simplified within a zero-order approximation

$$
\frac{\partial^{2} e^{-y} \Phi}{\partial y^{2}}=e^{-y} \Phi-2 e^{y} \frac{\partial \Phi}{\partial y}+e^{-y} \frac{\partial^{2} \Phi}{\partial y^{2}} \simeq \frac{\partial^{2} \Phi}{\partial y^{2}}
$$

Next, the zero-order boundary value problem is soluble with the help of a standard similarity transformation in terms of the variable $p=y / 2(\epsilon q)^{1 / 2}$. Equations (2.8) and (2.9) reduce to an ordinary differential equation

$\frac{d^{2} \Phi}{d p^{2}}=-2 p \frac{d \Phi}{d p}$ with $\Phi(-\infty)=0$ and $\Phi(+\infty)=1$

whose solution is

$$
\Phi(p)=\frac{1}{2}(1+\operatorname{erf} p) .
$$

We note that in principle Eq. (2.11) is satisfactory not too far outside the diffusive layer, i.e., for $|p| \ll \epsilon^{-1 / 2}$ otherwise Eq. (2.9) breaks down. However, although the approach to the respective limits 0 and 1 will certainly deviate from Eq. (2.11) well beyond this layer, the value of $\Phi$ itself turns out to be very well approximated by Eq. (2.11). A second potential difficulty is that Eq. (2.11) has a point of inflection at $p=0$ which is the center of an inner region where, again, Eq. (2.9) is poor. But a balance of terms in Eq. (2.9) using Eq. (2.11) shows that this region is of very small size $[O(\epsilon q)]$ and merely a fraction $\left[O\left(\epsilon^{1 / 2} q^{1 / 2}\right)\right]$ of the diffusive layer. Furthermore, the solution to Eq. (2.8) must increase monotonically so the influence of the inner region is negligible. Hence, we may regard Eq. (2.11) a 
uniformly valid approximation to the completc problem [Eq. (2.8)], provided $\epsilon \ll 1$. In terms of the original variables, Eq. (2.11) becomes

$$
\Theta \simeq \frac{e^{-\tau}}{2}\left[1+\operatorname{erf}\left(\frac{\log x-\tau}{2 \sqrt{\epsilon\left(1-e^{-\tau}\right)}}\right)\right] .
$$

This is fully consistent with Faxén's Eq. (2.5) throughout the diffusive layer within our zero-order approximation $\left[x=O(1), \tau=O(1),|y|=O\left(\epsilon^{1 / 2}\right), \log x=\tau\right.$ $+y$, see above]. Beyond the diffusive region Eqs. (2.5) and (2.12) all but coincide even though the respective asymptotic behaviors to the limits 0 and 1 are entirely different.

\section{SEDIMENTATION EQUATIONS FOR LINEAR MICELLES}

Suppose a solution of linear micelles is put in a nonequilibrium state characterized by, say, a weak concentration gradient of typical scale $\Lambda$. The original size distribution will readjust itself in two stages essentially. The first involves rearrangement of the surfactant molecules among the micelles because the latter break up and collide. We now assume that this process reaches local thermodynamic equilibrium (with respect to surfactant exchange) within a comparatively short time $\tau_{\text {loc }}$ and within a volume $r_{10 c}^{3} \ll \Lambda^{3}$; $r_{\text {loc }}$ and $\tau_{\text {loc }}$ are independent of $\Lambda$ if the latter is large enough. In the second stage, diffusion occurs on length scales $l_{d} \gg r_{\text {loc }}$ and time scales $\tau_{d} \gg \tau_{\text {loc }}$.

In view of these arguments, we introduce a hydrodynamic or coarse-grained description with all fields depending on the coordinates $\mathbf{r}$ and time $t$, i.e., point $\mathbf{r}$ refers to a box of dimension $v\left(r_{\text {loc }}^{3} \leqslant v \leqslant \Lambda^{3}\right)$ containing many micelles and $\delta t>\tau_{\text {loc }}$. Next, let $V$ be an arbitrarily chosen volume in the solution $(V \gg v)$. The total mole fraction of amphiphiles $V^{-1} \int_{V} X(\mathbf{r}, t) d \mathbf{r}$ within $V$ is not influenced by the exchange reactions. ${ }^{8}$ Hence, we can write the equation of continuity as

$$
\frac{\partial X(\mathbf{r}, t)}{\partial t}=-\frac{\partial}{\partial \mathbf{r}} \cdot \mathbf{J}_{\text {tot }}(\mathbf{r}, t),
$$

where the flux $J_{\text {tot }}(\mathbf{r}, t)$ defined with respect to a cell-fixed reference frame is

$$
\mathbf{J}_{\mathrm{tot}}(\mathbf{r}, t)=-k_{B} T \sum_{s>s_{0}} \frac{X_{s}(\mathbf{r}, t)}{\zeta(s)} \frac{\partial}{\partial \mathbf{r}} s \bar{\mu}(\mathbf{r}, t) .
$$

We have neglected cross terms in the mobility tensor and all intermicellar interactions (hydrodynamic or otherwise), thereby restricting ourselves to dilute solutions. $X_{s}(\mathbf{r}, t)$ is the mole fraction of amphiphiles in the state of aggregation indexed $s\left(X=\Sigma_{s>s_{0}} X_{s}\right), s_{0}$ is some minimum aggregation number, and $\xi(s)$ is the translational friction coefficient (averaged over all possible orientations) of a micelle containing $s$ amphiphiles. The chemical potential $\bar{\mu}$ (in units $k_{B} T \equiv \beta^{-1}$ ) of one amphiphile in a solution of linear micelles in local thermodynamic equilibrium is ${ }^{1}$

$$
\begin{aligned}
\bar{\mu}(\mathbf{r}, t)= & \mu_{0}\left(p_{\mathrm{ex}}, T\right)+K s^{-1}+s^{-1} \log X_{s}(\mathbf{r}, t) \\
& -n s^{-1} \log s+\beta m_{b} \Psi(\mathbf{r}),
\end{aligned}
$$

where $\mu_{0}$ is the chemical potential of one amphiphile in an infinitely long micelle in the absence of the field and depends on the external pressure $p_{\mathrm{ex}}$ and the temperature $T$, $K$ is the free energy of formation of two end caps, $n$ is a number $O(10)$ correcting for the loss of degrees of freedom of an amphiphile upon aggregation, $m_{b}$ is the mass of one amphiphile corrected for buoyancy, and $\Psi(r)$ is the potential of the centrifugal field which gives only an extensive contribution to the free energy of the system. ${ }^{1}$ The amphiphiles are supposed incompressible.

Defining $\eta(\mathbf{r}, t)$, which is assumed to be positive, as

$$
\eta(\mathbf{r}, t) \equiv \mu_{0}\left(p_{\mathrm{ex}}, T\right)+\beta m_{b} \Psi(\mathbf{r})-\vec{\mu}(\mathbf{r}, t)
$$

we may express the micellar distribution by

$$
X_{s}(\mathbf{r}, t)=s^{n} \exp [-s \eta(\mathbf{r}, t)-K]
$$

since $\bar{\mu}$ is independent of $s$ in local thermodynamic equilibrium. Expressions analogous to Eqs. (3.1) and (3.5) have appeared before in theories on micellar growth under stationary flow. ${ }^{3}$ What appears to be new is our focus on temporal phenomena. Ultimately, we wish to express all variables in terms of the mole fraction $X(\mathbf{r}, t)$ which can be measured in a sedimentation experiment. In the calculations below, we eliminate the function $\eta(r, t)$ with the help of the relation

$$
\begin{aligned}
X=\sum_{s>s_{0}} X_{s} & \simeq e^{-K} \int_{s_{0}}^{\infty} d s s^{n} e^{-s \eta} \\
& \simeq(2 \pi / n)^{1 / 2}(n / \eta)^{n+1} e^{-n-K} .
\end{aligned}
$$

The last equation follows from the Laplace method and the fact that $s_{0} \eta \ll n$.

Finally, we rewrite Eqs. (3.1) and (3.2) in the case of a centrifugal field $\Psi(\mathbf{r})=-\frac{1}{2} \omega^{2} r^{2}$ in cylindrical coordinates as

$$
\frac{\partial X}{\partial t}=\frac{1}{r} \frac{\partial}{\partial r}\left(k_{B} \operatorname{Tr} \frac{\partial \sigma_{0}}{\partial r}-m_{b} \omega^{2} r^{2} \sigma_{1}\right)
$$

with

$$
\begin{aligned}
& \sigma_{0}=\sigma_{0}(r, t) \equiv \sum_{s \gg s_{0}} \frac{X_{s}(r, t)}{\zeta(s)}, \\
& \sigma_{1}=\sigma_{1}(r, t) \equiv \sum_{s \geqslant s_{0}} \frac{s X_{s}(r, t)}{\zeta(s)} .
\end{aligned}
$$

The rest of this section is devoted to the evaluation of $\sigma_{0}$ and $\sigma_{1}$ in terms of $X$ for a number of specific models. In the absence of the external field, the reasoning presented above yields results similar to the diffusion equations derived by Cates et al. $^{8}$

\section{A. Dilute solutions of rodlike micelles}

The friction coefficient $\xi$ of a rod of length $L$ and diameter $d(L / d \gg 1)$ in a solvent of viscosity $\eta_{0}$ has the following form ${ }^{14-16}$

$$
\zeta=3 \pi \eta_{0} L[\log (L / d)+2 \log 2-1]^{-1} .
$$


The length $L$ of a rodlike micelle containing $s$ amphiphiles is given by $L=2 d\left(s-s_{c}\right) / 3 s_{c}$ where the constant $s_{c}$ denotes the number of amphiphiles within the two end caps. ${ }^{17}$ (We assume a uniform hydrocarbon density in the micellar core). For long micelles this can be simplified to $L \simeq 2 d s$ / $3 s_{c}$ for this is consistent with all previous approximations made.

For simplicity we set $n=1$ (which is often thought to be true). ${ }^{18}$ The functions $\sigma_{0}$ and $\sigma_{1}$ can now be calculated with the help of Eq. (3.6) and the following approximate expressions, valid for $s_{0} \eta \ll 1$ :

$$
\begin{aligned}
\int_{s_{0}}^{\infty} d s \log s e^{-s \eta} \simeq \frac{-\gamma-\log \eta}{\eta}, \\
\int_{s_{0}}^{\infty} d s s \log s e^{-s \eta} \simeq \frac{1-\gamma-\log \eta}{\eta^{2}},
\end{aligned}
$$

where $\gamma$ is Euler's constant.

$$
\begin{gathered}
\sigma_{0}=\frac{a_{1} X^{1 / 2} s_{c}}{\eta_{0} d e^{K / 2}}\left(\log X+K-2 \log s_{c}+a_{2}\right), \\
\sigma_{1}=\frac{a_{3} X s_{c}}{\eta_{0} d}\left(\log X+K-2 \log s_{c}+a_{4}\right),
\end{gathered}
$$

where the constants have the following values: $a_{1}$ $=\frac{1}{2}(2 \pi)^{-5 / 4} e^{1 / 2}, \quad a_{2}=2 \log \frac{8}{3}-1-2 \gamma-\frac{1}{2} \log 2 \pi, \quad a_{3}$ $=\frac{1}{2}(2 \pi)^{-3 / 2} e$, and $a_{4}=2 \log \frac{8}{3}+1-2 \gamma-\frac{1}{2} \log 2 \pi$.

\section{B. Dilute solutions of rodlike micelles in the free- draining limit}

Equations (3.7), (3.11), and (3.12) form a complicated problem tractable by tedious numerical analysis only. However, the logarithmic terms in Eqs. (3.11) and (3.12) vary within fairly narrow bounds. Accordingly, it is worthwhile to develop a free-draining approximation. We set

$$
\zeta(s)=\xi_{0} s
$$

with

$$
\zeta_{0} \equiv \frac{2 \pi \eta_{0} d}{s_{c}}\left[\log \left(L_{0} / d\right)+2 \log 2-1\right]^{-1}
$$

where $L_{0}$ is a zero-order estimate for the average length. After deriving $X(r, t)$ we calculate a new (spatial and time) average which forms the next iterate $L_{1}$, and so forth.

The functions $\sigma_{0}$ and $\sigma_{1}$ are evaluated as in Eq. (3.6). Next, Eq. (3.7) is rewritten using the transformations outlined in Sec. II

$$
\frac{\partial \Theta}{\partial \tau}=\frac{\partial}{\partial x}\left(\epsilon x \frac{\partial \Theta^{n /(n+1)}}{\partial x}-x \Theta\right),
$$

where $\tau \equiv 2 m_{b} \omega^{2} t / \zeta_{0}$

$$
\begin{aligned}
& \epsilon \equiv \frac{2 k_{B} T}{m_{b} \omega^{2} r_{0}^{2}} c(n) e^{-K /(n+1)} X_{0}^{-1 /(n \mid 1)} \\
& c(n) \equiv(2 \pi)^{1 /(2 n+2)} e^{1 /(n+1)} n^{\left(-2 n^{2}-n\right) /(2 n+2)}(n-1)^{n-1 / 2}
\end{aligned}
$$

$$
\text { for } n \neq 1 \text {, }
$$

and $c(1)=(2 \pi)^{-1 / 4} e^{1 / 2} \cdot c(n)$ changes monotonically from 0.6 for $n=2$ down to 0.4 for larger values of $n$. Typical values of the variables in Eq. (3.16) are: $K=30, m_{b}=5$ $\times 10^{-26} \mathrm{~kg}, \omega=6000 \mathrm{rad} / \mathrm{s}, r_{0}=0.07 \mathrm{~m}, T=298 \mathrm{~K}$, and $X_{0}=10^{-5}$, which give a value of $10^{-4}$ for $\epsilon$. Applying the approximation scheme introduced in the previous section, we arrive at the equivalent of Eq. (2.10) for rodlike micelles

$$
\begin{aligned}
\frac{d^{2} \Phi^{n /(n+1)}}{d p^{2}}= & -2 p \frac{d \Phi}{d p} \quad \text { with } \Phi(-\infty)=0 \\
& \text { and } \Phi(+\infty)=1
\end{aligned}
$$

with a different definition of $q: q \equiv(n+1) / n(1$ $\left.-e^{-n \tau /(n+1)}\right)$. In the next section we will fully investigate this nonlinear differential equation in the case $n=1$.

\section{Dilute solutions of linear flexible micelles}

Flexible micelles have a persistence length which is much smaller than the average contour length. However, they are generally quite slender (persistence length $\gg d$ ) so a simple Zimm model for the friction coefficient is often not applicable. Here, we simply set

$$
\zeta(s) \simeq \zeta_{p} s^{\nu},
$$

where $\zeta_{p}$ and $v$ are empirical constants. Using Eq. (3.18) to calculate $\sigma_{0}$ and $\sigma_{1}$ and again taking $n=1$ for simplicity, we have

$$
\begin{aligned}
& \sigma_{0}=\frac{(2 \pi)^{v / 4}}{\zeta_{p}} e^{\nu(-K+1) / 2}(1-v)^{-v+3 / 2} X^{1-v / 2}, \\
& \sigma_{1}=\frac{(2 \pi)^{(v-1) / 4}}{\zeta_{p}} e^{(K-1)(1-v) / 2}(2-v)^{-v+5 / 2} X^{(3-v) / 2}
\end{aligned}
$$

Hence, Eq. (3.7) is now a generalized Burgers equation ${ }^{19}$ where both terms on the right-hand side contain rational powers of $X$.

\section{ANALYSIS OF EQUATION (3.17)}

We analyze and solve Eq. (3.17) numerically in the case $n=1$. After substituting $Y(p) \equiv \Phi(p)^{1 / 2}$ we get

$\frac{d^{2} Y}{d p^{2}}=-4 p Y \frac{d Y}{d p}$ with $Y(-\infty)=0$ and $Y(+\infty)=1$.

It can be proved that this equation does not belong to any of the 50 classes of second-order nonlinear ordinary differential equations distinguished by Painlevé. ${ }^{20}$ Equation (4.1) is therefore nontrivial for it contains spontaneous or movable singularities.

We first investigate the limiting behavior of $Y(p)$ as $p \rightarrow+\infty$, in which case linearization of Eq. (4.1) is possible. We set

$$
Y(p)=1-\epsilon(p), \quad \epsilon(p) \ll 1 \quad \text { as } p \rightarrow+\infty .
$$

Substitution of Eq. (4.2) in Eq. (4.1) and keeping terms of linear order in $\epsilon$ yield the equation 


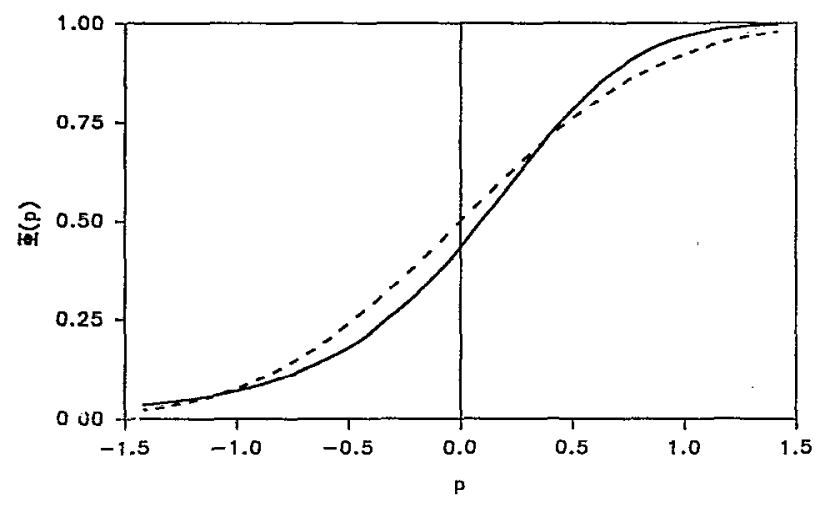

FIG. 3. Plot of the numerical calculation of $\Phi(p)$ for rodlike micelles in the free-draining limit. The symmetric plot (dashed curve) holds for a nonaggregating solute.

$$
\epsilon^{\prime \prime}(p)=-4 p \epsilon^{\prime}(p) \text { with } \epsilon(+\infty)=0
$$

whose solution is

$$
\epsilon(p)=c_{1}\left(1-\operatorname{erf} 2^{1 / 2} p\right) .
$$

Having established Eq. (4.4), we next consider it convenient to solve Eq. (4.1) numerically by using a fourthorder Runge-Kutta scheme with Eq. (4.2) as input. ${ }^{21} \mathrm{We}$ have determined $c_{1}$ to be 0.3816 by minimizing $Y(p=$ $-1000)$. The numerical solution is plotted in Fig. 3.

At this stage it becomes sensible to address the limiting behavior of $Y$ as $p \rightarrow-\infty$. First it is noted that Eq. $(4: 1)$ is scale invariant. Next, substitution of $Y(p) \equiv[u(p)] / p^{2}$ and $p \equiv-e^{t}$, leaves us with an autonomous differential equation

$$
\frac{d^{2} u}{d t^{2}}-5 \frac{d u}{d t}+6 u=-4 u \frac{d u}{d t}+8 u^{2}
$$

Inspection of our numerical results urges us to surmise that $u(t) \sim 3 / 4$ for $t \rightarrow \infty$. Thus we tentatively set

$$
u(t)=3 / 4-\omega(t), \quad \omega(t) \ll 1 \quad \text { as } t \rightarrow \infty .
$$

After substituting this in Eq. (4.5) and retaining terms of linear order in $\omega$, we obtain an Euler equation whose solution leads to

$$
u(p) \sim 3 / 4-c_{2}(-p)^{1-\sqrt{7}} \text { as } p \rightarrow-\infty .
$$

Our numerical calculations do indeed agree completely with this limiting form; the constant $c_{2}$ equals 1.24.

The analytical structure of Eqs. (4.4) and (4.7) shows that the deceptively simple looking Eq. (4.1) is difficult to solve, even approximately. We have made little or no attempt to determine a globally valid approximation.

\section{CONCLUDING REMARKS}

The theory presented here is valid only for dilute solutions of linear aggregates. Extenision to the nondilute case would require careful scrutiny of a variety of correction terms. We stress that the treatment is definitely nonrigorous. For instance, we have not discussed precisely what happens close to zero concentration at the top of the cell. Then, the micelles are either spherical or almost nonexist- ent so Eq. (3.2) is no longer correct. However, the extent of this anomalous region is negligible when the micelles are sufficiently slender at the mole fraction $X_{0}$.

Besides the approximations emphasized earlier, we also point out that rodlike micelles are not absolutely rigid but inevitably wormlike though the undulations may be small. This effect introduces yet another perturbative nonlinearity in the continuity equation. On the whole, a rigorous mathematical justification for our physically motivated self-similar solution of the first kind would be very complicated and is outside the scope of this work. Nor have we discussed the sedimentation of flexible micelles in more detail, for we suspect the solution to the continuity equation is again self-similar but of an entirely different structure, namely of the second kind with an associated nonlinear eigenvalue problem. ${ }^{19}$ An exhaustive analysis of this entails a paper by itself.

In the usual centrifuge experiment of sedimenting particles that do not aggregate, the scaled concentration profile is symmetric (Fig. 3 ) in the intermediate regime because the transport equation is linear [Eq. (2.1)]. By contrast, for rodlike aggregates the profile is asymmetric (Fig. 3) since the transport equation is no longer linear [Eqs. (3.5) and (3.7) are coupled]. In the free-draining limit, the centrifugal driving force on a micelle is exactly proportional to the friction on it exerted by the solvent so the driving term $\sigma_{1}$ is proportional to the volume fraction as it should be. But the diffusion term $\sigma_{0}$ is nonlinear [in fact proportional to $X^{1 / 2}$; see Eq. (3.11)] in view of the restriction given by Eq. (3.5) signifying the local equilibrium of the scission and recombination kinetics of the micelles. The diffusion coefficient thus scales as $X^{-1 / 2}$ and such a diffusion process is termed "fast." 19 This terminology is reasonable from a physical point of view: in the top of the cell, the surfactant concentration is low and the micelles are generally fairly small so they diffuse faster than aggregates in the bottom part of the cell. However, it is difficult to understand the complete structure of the asymmetric curve in Fig. 3 for reasons stated in the previous section.

Finally, we illustrate how a zero-order free-draining approximation may be applied to the characterization of rodlike micelles by dynamic centrifugation. For simplicity, we discuss the case $n=1$ only. First, the mole fraction of surfactant $X$ is determined as a function of distance $r$ and time $t$ in the intermediate region (Fig. 2). If the centrifuge rotates fast enough, the diffusive boundary layer will be relatively thin at all times i.e., $\epsilon \ll 1$, so it should be possible to distinguish a sedimenting front. Ideally, the front should move in such a way that $z_{f} \equiv 2 \log \left(r_{f} / r_{0}\right)$ is linear in $t$; the slope $2 m_{b} \omega^{2} / \xi_{00}$ yields the friction coefficient $\xi_{00}$ in the zero-order free-draining approximation. Next, the diffusive layer is analyzed on a much finer scale $z \equiv 2 \log \left(r / r_{0}\right)$ at various scaled times $\tau \equiv 2 m_{b} \omega^{2} t / \xi_{00}$. Plots of the variable $\Phi \equiv e^{\tau} X / X_{0}$ as a function of $(z-\tau) / q^{1 / 2}$ with $q \equiv 2(1$ $-e^{-\tau / 2}$ ) should collapse onto one curve. Comparison of this universal plot with Fig. 3 gives the sought after quantity $\epsilon \equiv 2 e^{1 / 2} k_{B} T /(2 \pi)^{1 / 4} m_{b} \omega^{2} r_{0}^{2} e^{K / 2} X_{0}^{1 / 2}$ via the identity $p \equiv(z-\tau) / 2 \epsilon^{1 / 2} q^{1 / 2}$. In this way we obtain the end cap 
term $K$ and a zero-order friction coefficient $\xi_{00}$. The iterative free-draining scheme outlined in Sec. III takes the hydrodynamic interaction into account to some extent but is much more elaborate.

\section{ACKNOWLEDGMENTS}

We thank Dr. M. R. Hardeman and P. T. Goedhart for a discussion on the rheology of blood suspensions.

${ }^{1}$ A. Duyndam and T. Odijk, Langmuir 9, 1160 (1993).

${ }^{2}$ M. E. Cates, Macromolecules 20, 2289 (1987).

${ }^{3}$ M. S. Turner and M. E. Cates, J. Chem. Soc. Faraday Trans. 87, 2073 (1991).

${ }^{4}$ M. S. Turner and M. E. Cates, J. Phys.: Condens. Matter 4, 3719 (1992).

${ }^{5}$ S. Q. Wang, W. M. Gelbart, and A. Ben-Shaul, J. Phys. Chem. 94, 2219 (1990).

${ }^{6}$ S. Q. Wang, J. Phys. Chem. 94, 8381 (1990).

${ }^{7}$ R. Bruinsma, W. M. Gelbart, and A. Ben-Shaul, J. Chem. Phys. 96, 7710 (1992).

${ }^{8}$ M. E. Cates, C. M. Marques, and J..P. Bouchaud, J. Chem. Phys. 94, 8529 (1991).

${ }^{9}$ H. Hoffmann, J. Kalus, H. Thurn, and K. İbel, Ber. Bunsenges. Phys. Chem. 87, 1120 (1983).

${ }^{10} \mathrm{~J}$. Kalus, H. Hoffmann, K. Reizlein, W. Ulbricht, and K. Ibel, Ber. Bussenges. Phys. Chem. 86, 37 (1982).
${ }^{11}$ H. Fujita, Mathematical Theory of Sedimentation Analysis (Academic, New York, 1962).

${ }^{12}$ H. Fujita, Foundations of Ultracentrifugal Analysis (Wiley, New York, 1975).

${ }^{13}$ W. J. Archibald, Ann. N.Y. Acad. Sci. 43, 211 (1942).

${ }^{14}$ H. Yamakawa and M. Fujii, Macromolecules 6, 407 (1973).

${ }^{15}$ T. Yoshizaki and H. Yamakawa, J. Chem. Phys. 72, 57 (1980).

${ }^{16}$ H. Yamakawa, Ann. Rev. Phys. Chem. 35, 23 (1984).

${ }^{17}$ P. J. Missel, N. A. Mazer, G. B. Benedek, C. Y. Young, and M. C. Carey, J. Phys. Chem. 84, 1044 (1980).

${ }^{18}$ J. N. Israelachvili, D. J. Mitchell and B. W. Ninham, J. Chem. Soc. Faraday Trans. 2 72, 1525 (1976).

${ }^{19}$ P. L. Sachdev, Nonlinear Diffusive Waves (Cambridge University, Cambridge, UK, 1987).

${ }^{20}$ Following Ince's scheme in determining the necessary and sufficient conditions for the absence of movable singularities we see that Eq. (4.1) belongs to subcase $i$ (b) in paragraph 14.312 of E. L. Ince, Ordinary Differential Equations (Dover, New York, 1956), using the transformation $\lambda(z)=1 / 2 z$. After the more general transformation $w=(2 z)^{-1} W+\mu(z)$ and $z=\phi(z)$, the resulting equation should not have a tcrm proportional to $W$ thus restricting $\mu(z)$ to $\mu=\left(1 / 6 z^{2}\right)+c z$ where $c$ is an arbitrary constant. Then, the functions $P(z)$ and $Q(z)$ defined by Ince are not identical which is necessary for the general solution of Eq. (4.1) to be free from movable singularities.

${ }^{21}$ M. Abramowitz and I. A. Stegun, Handbook of Mathematical Functions (Dover, New York, 1968). 